\title{
Distorted Wave Theories Applied to Double Ionization by Ion Impact: Simulation of Higher-Order Processes
}

\author{
M. F. Ciappina, ${ }^{1}$ T. Kirchner, ${ }^{2}$ M. Schulz, ${ }^{3}$ D. Fischer, ${ }^{4}$ R. Moshammer, ${ }^{4}$ and J. Ullrich ${ }^{4}$ \\ ${ }^{1}$ Institute of High Performance Computing, 1 Fusionopolis Way, \#16-16 Connexis, 138632, Singapore \\ ${ }^{2}$ Department of Physics and Astronomy, York University, 4700 Keele Street, Toronto, ON, Canada M3J 1P3 \\ ${ }^{3}$ Department of Physics and Laboratory for Atomic, Molecular, and Optical Research, Missouri University of Science and Technology, \\ Rolla, MO 65409, USA \\ ${ }^{4}$ Max-Planck-Institut für Kernphysik, Saupfercheckweg 1, 69117 Heidelberg, Germany
}

Correspondence should be addressed to T. Kirchner, tomk@yorku.ca

Received 14 January 2010; Accepted 24 March 2010

Academic Editor: Igor Bray

Copyright (C) 2010 M. F. Ciappina et al. This is an open access article distributed under the Creative Commons Attribution License, which permits unrestricted use, distribution, and reproduction in any medium, provided the original work is properly cited.

\begin{abstract}
One of the goals in studies of double ionization (DI) of simple atoms by electron or ion impact is to elucidate and assess the different mechanisms that lead to this atomic process. In this work we present an attempt to model the mechanisms beyond the first order in DI of helium by highly charged projectiles. To this end we employ the continuum distorted wave-eikonal initial state (CDW-EIS) formalism joint with a Monte Carlo event generator (MCEG). The MCEG allows us to generate theoretical event files that represent the counterpart of the data obtained from a kinematically complete experiment. Starting from these event files, a new data analysis tool used to contrast theory and experiment in DI, the four-body Dalitz plots, is easily produced. The higher order mechanisms are simulated by considering DI as a sequential process: a single ionization of a helium atom as a first step and another single ionization of a single-charged helium ion as a second step. Some of the features in the experimental data are very well reproduced by these simulations.
\end{abstract}

\section{Introduction}

Among the atomic breakup processes, double ionization (DI) of simple atoms by charged particles represents a particularly interesting case to study the few body dynamics. The main difference between DI and its simplest counterpart, single ionization (SI), is the introduction of an ingredient that plays an important role under certain kinematics conditions: the electron-electron correlation. Additionally, DI proceeds by different pathways, and one of the goals of the theory is to assess the role of each of them for a given set of the parameters. It is common to consider that the total cross section for DI is mainly dominated by three different mechanisms. Two of them are first-order mechanisms and the third one is a higher-order mechanism. The two firstorder processes are the two-step-one projectile-electron interaction (TS-1) and the so-called shake-off (SO). In TS-1 only one electron is released through a direct interaction with the incoming projectile. This ejected electron then collides in the second step with the other electron liberating it to the continuum. The SO is very similar to TS- 1 in that only one electron is ejected by a direct interaction with the projectile. However, here the second electron gets ejected through rearrangement of the electronic wave function adjusting to a new potential resulting from the ejection of the first electron. Both TS-1 and SO mechanisms are first-order processes in the projectile-target atom interaction and in both some form of electron-electron correlation is required for DI to occur. For simplicity in the following we refer to both of them as TS-1. Finally, in the third mechanism, dubbed two-steptwo projectile-electron interaction (TS-2), both electrons are ejected through two independent interactions with the projectile. This is a higher-order process which does not require neither does it rule out electron-electron correlation [1].

Recently, it was demonstrated that a pure form of TS-1 is never realized [2]. Previously, it was assumed that TS- 1 is dominant in very fast collisions (except for highly charged 
ions) and TS-2 for slow collisions and/or highly charged ion impact. However, later it was demonstrated that even for very fast proton impact the most important DI mechanism is a hybrid process between TS- 1 and TS-2. This channel, called TS-1-EL, can be viewed as a TS-1 process followed by elastic scattering between the projectile and one of the electrons already ejected to the continuum.

In recent years there has been a steady advance of experimental techniques in atomic physics. Nowadays kinematically complete experiments on diverse fragmentation processes are feasible, for example, single and double ionizations by charged particle impact [3-8] or mutual ionization of both collision partners [9]. Especially Cold Target Recoil Ion Momentum Spectroscopy (COLTRIMS), also known as Reaction Microscopes, has opened a new era of atomic collision experiments [10]. The data obtained from such experiments are very complex with several measured quantities being afflicted with experimental resolution and acceptance limitations. To fully account for these experimental restrictions in theoretical calculations, Monte Carlo Event Generators (MCEGs) are a very powerful tool.

In atomic collisions, MCEG have been developed and applied only recently [11]. In further developments, MCEG have been extended to treat DI of helium and also were used in order to analyze the DI with a new and powerful analysis tool: the four-body Dalitz plots (4D) [12]. In a kinematically complete experiment, the cross sections are usually extracted from a data file known as event file. Such file contains all three momentum components of all collision fragments recorded for a large number of fragmentation events. Theoretical approaches usually do not provide such an event file obtained in experiment. Rather, the probability that an event with a certain combination of momentum components of the collision fragments occurs is computed directly. An important shortcoming of this approach is that this makes a direct comparison between measured data and theory accounting for the experimental conditions difficult. As long as the cross sections depend only on one or two quantities which are afflicted by experimental resolution, this can be accounted for by analytically convoluting the calculation with the resolution. However, fully differential cross sections (FDCSs) can depend on a large number of parameters and a corresponding analytic convolution is simply not feasible. A convolution by numerical integration is usually not a realistic option either because it would make the computation prohibitively time-consuming. These problems can be avoided if an event file with the same structure as the one obtained in the experiment is generated using a certain theoretical approach. The experimental resolution can then be accounted for by adding eventby-event a random number, distributed according to the spectrometer function of the apparatus (typically a Gauss distribution), to each momentum component [11].

Another important advantage of using a theoretical event file is that additional physics, which may be problematic to accurately account for in the calculation, can be added retroactively. For example, the interaction between the nuclei of both collision partners (NN interaction) was often neglected in previous studies. However, convoluting the results of theory with classical elastic scattering between the nuclei using the MCEG technique was recently demonstrated to yield much better agreement between experiment and theory for single ionization in fast $\mathrm{C}^{6+}+\mathrm{He}$ impact [13] and intermediate energy $p+\mathrm{H}$ collisions [14].

Finally, one of the most important benefits of employing theoretical event files is the capability of computing cross sections which are numerically too intensive using conventional methods. Usually, theoretical cross sections are obtained by appropriate integration of the FDCS. For example, double ionization entails 8 independent momentum components (12 components of the 4 final-state particles minus 4 kinematic conservation laws). The computation of a single differential cross section thus requires a sevenfold integration. For sophisticated theoretical models to a large extent this integration often has to be done numerically. However, the symmetry of the problem can considerably reduce the dimensionality of the integration so that the computation of the single differential cross section is not too problematic. On the other hand, if the cross section of interest only entails limited symmetry, or none at all, the numeric efforts required by the integration can quickly make the calculation unfeasible. Using an event file, in contrast, any cross section measured in the experiment can be obtained simply by sorting the histograms precisely the same way as done for the measured data (for computational details see [15]). A prominent example is the so-called fourparticle Dalitz (4-D) plots [12], as mentioned above.

The successful usage of these new tools requires reliable and robust theories to account for the underlying ionization dynamics. Over the years continuum distorted wave-basedformalisms have been used extensively for single ionization by electron and ion impact (see, e.g., [16] for a recent review), but, to the best of our knowledge, there is no such approach for double ionization due to the difficulties present to develop a formal theory beyond the First Born approximation. Although the TS-2 simulations do not represent a rigorous continuum distorted wave theory for DI, we demonstrate that nevertheless important qualitative information can be extracted from a comparison with measured data.

The article is organized as follows. The next section describes briefly the theory behind the TS-2 simulations and the computational tools used to produce them. In Section 3 we present a comparison between our theory and experiment. Specifically, we investigate the sensitivity of the theoretical results with respect to the simulation of elastic scattering between the heavy particles. Conclusions and an outlook are offered in Section 4. Atomic units are used throughout the article unless otherwise stated.

\section{The Monte Carlo Event Generator (MCEG) and the TS-2 Simulations}

As a first attempt to simulate the TS-2 mechanisms we employ the Monte Carlo Event Generator (MCEG) [11] along with the CDW-EIS theory [17]. The MCEG allows us to generate a theoretical event file that will be later used to produce the 4-body Dalitz (4-D) plots $[2,12,18,19]$. 
The outcome of the MCEG is a data file, based on a calculation of FDCS for the process of interest (see [15] for computational details). These FDCSs are computed using the CDW-EIS approach which takes into account higher-order contributions to the ejection of each electron.

In double ionization (DI), the number of momentum components per event contained in the event file depends both on the theoretical scheme we apply and the type of processes we want to model, namely, first-order processes or higher-order mechanisms. We model the TS-2 process as a combination of two independent SI steps. Consequently the event file contains the independent momentum components of both SI steps, namely, $\left(k_{1, x}, k_{1, y}, k_{1, z}, q_{1, x}\right)$ for the first SI step, that is, the single ionization of a neutral helium atom, and $\left(k_{2, x}, k_{2, y}, k_{2, z}, q_{2, x}\right)$ for the second step, that is, the single ionization now of an $\mathrm{He}^{+}$ion. Here $\left(k_{i, x}, k_{i, y}, k_{i, z}\right)$, with $i=$ 1,2 , are the cartesian components of the electron momentum and $\left(q_{i, x}, 0\right)$ the single component of the transverse momentum transfer for each SI step.

In order to produce the 4-D plots for DI of helium by ion impact, typically a few 100,000 events are required. A summary of the procedure followed to generate a theoretical event file will be presented below. The described cycle is repeatedly executed until the number of events needed is reached.

(i) A set of ten random numbers is chosen, eight of which represent the final-state momentum components of the fragments for which the FDCS will be calculated, that is, $k_{1, x}, k_{1, y}, k_{1, z}, q_{1, x}, k_{2, x}$, $k_{2, y}, k_{2, z}, q_{2, x}$. The ranges of each electron momentum component and of the momentum transfer components, that is, the intervals of the corresponding random number distributions, are established in advance according to the experimental conditions present in the particular DI process. The ninth and tenth quantities, $u$ and $w$, are random numbers uniformly distributed in the interval $[0,1]$.

(ii) The FDCSs are calculated at the randomly selected point. For the case of TS-2 simulation, we employ in each SI step the CDW-EIS model of Crothers and McCann [17, 20]. This model represents one of the most successful theories for SI of atoms by single and multiply charged ion impact in the range of intermediate to large projectile energies [21]. Since our aim is to model higher-order processes, the CDW-EIS appears to be the most adequate choice.

(iii) Once the FDCSs for each SI steps are obtained from the CDW-EIS model, the randomly selected event is considered valid and stored if both of the following conditions are fulfilled

$$
\begin{gathered}
u<\tilde{\sigma}_{\mathrm{SI}}, \\
w<\tilde{\sigma}_{\mathrm{SI}^{+}},
\end{gathered}
$$

otherwise the event is discarded. $\tilde{\sigma}_{\mathrm{SI}}\left(\widetilde{\sigma}_{\mathrm{SI}^{+}}\right)$corresponds to the normalized cross sections for SI of neutral helium $\left(\mathrm{He}^{+}\right.$ ion) calculated using $\widetilde{\sigma}_{\mathrm{SI}}=\sigma_{\mathrm{SI}} / \sigma_{\mathrm{SI}}^{\max }\left(\widetilde{\sigma}_{\mathrm{SI}^{+}}=\sigma_{\mathrm{SI}^{+}} / \sigma_{\mathrm{SI}^{+}}^{\max }\right)$ where $\sigma_{\mathrm{SI}}^{\max }\left(\sigma_{\mathrm{SI}^{+}}^{\max }\right)$ is the maximum value of the cross section within the selected phase space. As the cross section for both SI steps drop rapidly towards larger values of electron momenta and momentum transfers, the simple rejection method described above becomes quite inefficient and consequently many events are rejected so that the cycles (i)-(iii) have to be repeated hundreds of millions or billions of times. Fortunately, using a simple parallelization scheme, we can take advantage of the independence of this kind of Monte Carlo-based calculations, in the sense that the momentum space can be sampled independently and in this way a concurrent computation with high efficiency is easily implementable (see [15] for further details).

The FDCSs of each SI, that is,. $\sigma_{\mathrm{SI}}$ and $\sigma_{\mathrm{SI}^{+}}$, are calculated using the CDW-EIS approach of Crothers and McCann [17] and can be written as

$$
\sigma_{\mathrm{SI}, \mathrm{SI}^{+}}=\frac{\mathrm{d}^{5} \sigma}{\mathrm{d} \mathbf{k d} \mathbf{q}_{\perp}} \propto\left|T_{i f}^{-\mathrm{CDW}-\mathrm{EIS}}\right|^{2} \delta\left(E_{f}-E_{i}\right),
$$

where we have ignored the constants because they cancel out during the normalization procedure explained above. In (2), the electron momentum is $\mathbf{k}=\left(k_{i, x}, k_{i, y}, k_{i, z}\right)$ and the transverse momentum transfer $\mathbf{q}_{\perp}=\left(q_{i, x}, 0\right)$ with $i=1,2$ for each SI step. We employ nonorthogonal Jacobi coordinates $\left(\mathbf{r}_{P}, \mathbf{r}_{T}\right)$ to describe the collision process. These coordinates represent the position of the active electron of the neutral helium (or the single electron of the $\mathrm{He}^{+}$ion) with respect to the projectile $\left(\mathbf{r}_{P}\right)$ and the target ion $\left(\mathbf{r}_{T}\right)$, respectively. $\mathbf{R}_{T}$ is also needed, representing the position of the incoming ion with respect to the $\mathrm{CM}$ of the subsystem e-T. Neglecting terms of orders $1 / M_{T}$ and $1 / M_{P}$, where $M_{T}$ is the mass of the target ion nucleus and $M_{P}$ is the corresponding mass of the incident heavy ion, we can write $\mathbf{R}_{T}=\mathbf{r}_{T}-\mathbf{r}_{P}$ and in this way the transition matrix $T_{i f}^{- \text {CDW-EIS }}$ is separable in the variables $\mathbf{r}_{P}$ and $\mathbf{r}_{T}$ as it will become apparent below.

Within prior CDW-EIS, the transition amplitude can be computed as

$$
T_{i f}^{-\mathrm{CDW}-\mathrm{EIS}}=\left\langle\chi_{f}^{-\mathrm{CDW}}\left|W_{i}\right| \chi_{i}^{+\mathrm{EIS}}\right\rangle,
$$

where the initial (final) state distorted wave $\chi_{i}^{+}\left(\chi_{f}^{-}\right)$is an approximation to the initial (final) state which satisfies outgoing-wave $(+)$ (incoming-wave $(-)$ ) boundary conditions. For the initial state, the asymptotic form of the Coulomb distortion (eikonal phase) is used in the electron-projectile interaction. To model the initial boundstate wavefunction, we employ a semianalytical RoothaanHartree-Fock for the description of the neutral helium atom [22] and a hydrogenic 1s state with $Z=2$ for the $\mathrm{He}^{+}$ion. Consequently, the full initial state $\chi_{i}^{+\mathrm{EIS}}$ of (3) can be written as

$$
\chi_{i}^{+\mathrm{EIS}}=(2 \pi)^{-3 / 2} \exp \left(\mathrm{iK}_{i} \cdot \mathbf{R}_{T}\right) \psi_{\mathrm{He}, \mathrm{He}}\left(\mathbf{r}_{T}\right) \boldsymbol{E}_{v}^{+}\left(\mathbf{r}_{P}\right),
$$

where $\mathcal{E}_{v}^{+}\left(\mathbf{r}_{P}\right)$ is

$$
\mathcal{E}_{v}^{+}\left(\mathbf{r}_{P}\right)=\exp \left(-\mathrm{i} \frac{Z_{P}}{v} \ln \left(v r_{P}-\mathbf{v} \cdot \mathbf{r}_{P}\right)\right),
$$




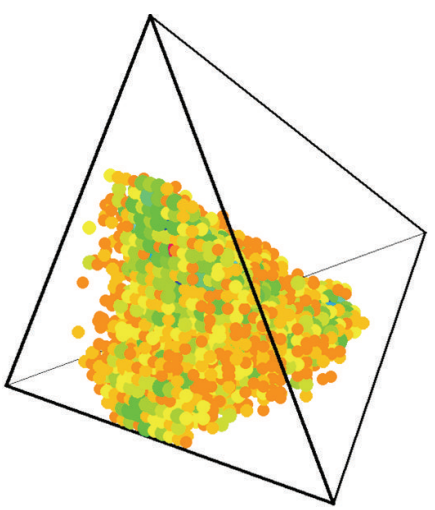

(a)

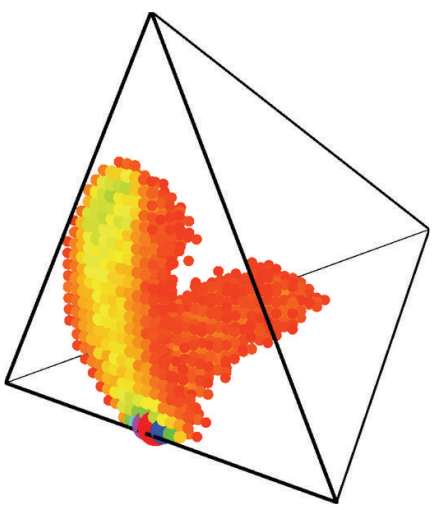

(c)

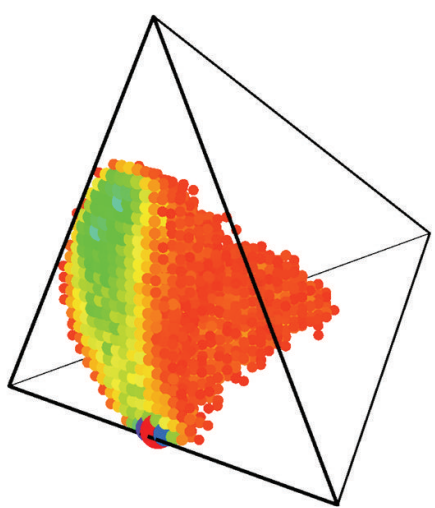

(b)

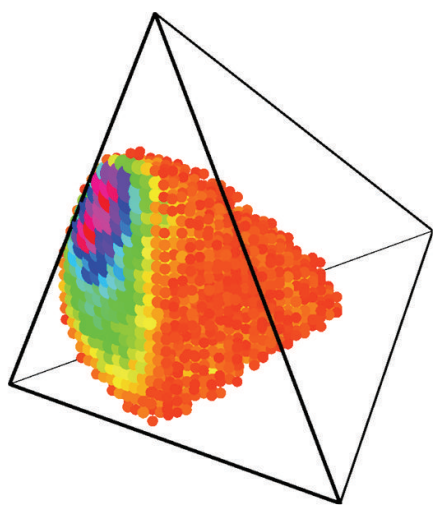

(d)

Figure 1: (Color online) Four body Dalitz plots (4-D) for DI in $158 \mathrm{MeV} \mathrm{Au}{ }^{33+}+\mathrm{He}$ collisions. (a) experimental data, (b)-(d) TS-2 CDWEIS for different values of $b P(b)$ (see text for details).

and $\psi_{\mathrm{He}_{3} \mathrm{He}^{+}}\left(\mathbf{r}_{T}\right)$ is the initial bound state of the neutral helium atom or the $\mathrm{He}^{+}$ion.

The final state wavefunction is cast into the form [23]

$$
\chi_{f}^{-\mathrm{CDW}}=(2 \pi)^{-3 / 2} \exp \left(\mathrm{i} \mathbf{K}_{f} \cdot \mathbf{R}_{T}\right) \chi_{T}^{-}\left(\mathbf{r}_{T}\right) C_{P}^{-}\left(\mathbf{r}_{P}\right),
$$

where $C_{P}^{-}$represents the Coulomb distortion of the ejected electron wave function due to the projectile.

$$
C_{P}^{-}\left(\mathbf{r}_{P}\right)=N\left(\nu_{P}\right){ }_{1} \mathrm{~F}_{1}\left(-\mathrm{i} \nu_{P}, 1,-\mathrm{i} k_{P} r_{P}-\mathrm{i} \mathbf{k}_{P} \cdot \mathbf{r}_{P}\right),
$$

where $v_{P}=Z_{P} / k_{P}$ is the Sommerfeld parameter, $\mathbf{k}_{P}$ is the relative momentum of the e-P subsystem and $N\left(\nu_{P}\right)$ is the usual Coulomb factor

$$
N\left(\nu_{P}\right)=\Gamma\left(1-\mathrm{i} \nu_{P}\right) \exp \left(\frac{\pi \nu_{P}}{2}\right)
$$

On the other hand, $\chi_{T}^{-}\left(\mathbf{r}_{T}\right)$ is the wave function for the ejected electron in the field of the target residual ion.

$$
\begin{aligned}
\chi_{T}^{-}\left(\mathbf{r}_{T}\right)= & (2 \pi)^{-3 / 2} \exp \left(\mathrm{i} \mathbf{k}_{T} \cdot \mathbf{r}_{T}\right) N\left(\nu_{T}\right) \\
& \times{ }_{1} \mathrm{~F}_{1}\left(-\mathrm{i} \nu_{T}, 1,-\mathrm{i} k_{T} r_{T}-\mathrm{i} \mathbf{k}_{T} \cdot \mathbf{r}_{T}\right)
\end{aligned}
$$

with $\nu_{T}=Z_{T} / k_{T}$ and where $\mathbf{k}_{T}$ is the relative momentum of the e-T subsystem. For the neutral helium atom, we use an effective charge $Z_{T}=1.69$ in order to consider the partial screening of the passive electron. On the other hand, for the SI of the $\mathrm{He}^{+}$ion, $Z_{T}=2$ is employed.

The perturbation potential $W_{i}$ in (3) is defined by [24]

$$
\left(H_{i}-E_{i}\right) \chi_{i}^{+}=W_{i} \chi_{i}^{+},
$$

where $H_{i}$ is the full electronic initial Hamiltonian (neglecting the total CM motion) and $E_{i}$ is the total initial energy of the system in the CM frame, respectively. $W_{i}$ is composed by two differential operators that can be written

$$
W_{i}=\frac{1}{2} \nabla_{\mathbf{r}_{P}}^{2}-\nabla_{\mathbf{r}_{T}} \cdot \nabla_{\mathbf{r}_{P}}
$$

In the computation of the FDCS (2), we have neglected the interaction between the heavy nuclei, the so-called NN interaction. This interaction, however, is incorporated later in the processing of the event file (see, e.g., [13] for details) and is taken into account in our simulations.

\section{Results and Discussions}

In Figures 1-3, 4-D plots are shown for DI in $158 \mathrm{MeV}$ $\mathrm{Au}^{33+}+\mathrm{He}$ collisions. Similar plots were discussed in [19], but here we place emphasis on an analysis of the sensitivity of the theoretical results with respect to the simulation of elastic scattering between the heavy particles. Details on how 


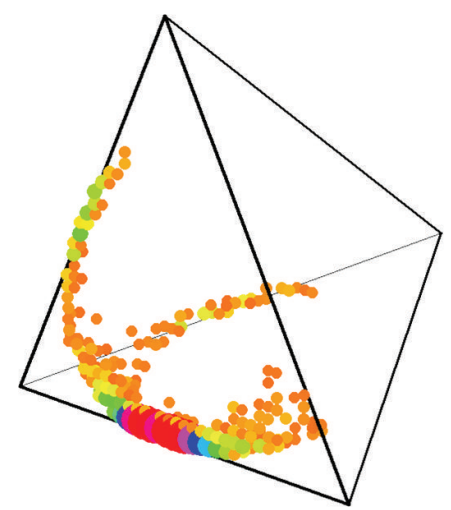

(a)

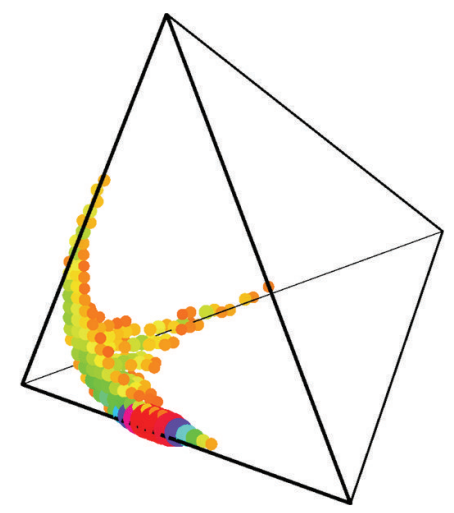

(c)

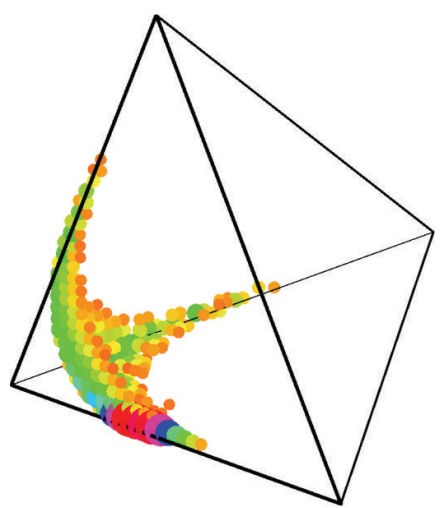

(b)

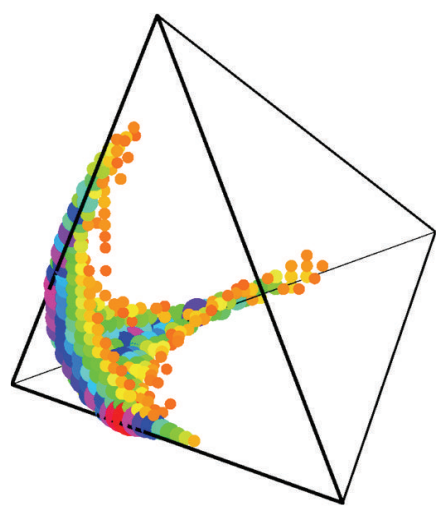

(d)

Figure 2: (Color online) Same as Figure 1 for the transverse momentum components of each particle in the scattering plane (see the text for details).

to read 4-D plots were presented previously $[12,15,18]$. In short, a tetrahedral coordinate system is employed where each tetrahedron plane represents one of the four final-state fragments of the collision (front and bottom planes: ejected electrons; backplane: target nucleus; right plane: momentum transfer from projectile to target atom). The distance of a given data point to the four planes represents a set of the relative squared momenta of the final-state particles $\pi_{i}=p_{i}^{2} / \sum p_{j}^{2}$. In Figure 1, the comparison between the experimental (panel a) and theoretical 4-D plots, calculated within the TS-2 CDW-EIS model (panel b), shows only marginal, qualitative agreement as far as the boundary of the area containing any data points at all is concerned. However, the intensity distribution within these boundaries is poorly reproduced. This lack of agreement is not entirely surprising considering the very large perturbation parameter $\eta$ (projectile charge to speed ratio) of 5.8. For such extreme $\eta$, the cross sections are dominated by higher-order contributions which are extremely difficult to accurately account for theoretically. It is not even clear whether a perturbative expansion still converges. As a result, the calculations become very sensitive to the details of the description of the various interactions. A simple example is illustrated in panels (b) through $(\mathrm{d})$ in Figure 1. There, different assumptions were made for $b P(b)$ for double ionization (where $b$ is the impact parameter and $P(b)$ is the double ionization probability), which enter in the convolution of the FDCS with elastic scattering between the heavy particles [13]. In panel (b), $b P(b)$ was assumed to maximize at $b=10$ a.u., in panel (c) at $b=6$ a.u., and in panel (d) the FDCSs were not convoluted at all with elastic scattering. The significant qualitative changes in the corresponding 4-D plots are quite apparent.

In Figure 2 experimental and theoretical 4-D plots are shown for the same collision system and in the same order as in Figure 1. However, this time only the transverse momentum components of each particle in the scattering plane (spanned by the initial and final projectile momenta) were used in the computation of the $\pi_{i}$. Remarkably, in this case the experimental data are reasonably well reproduced by theory. This improved agreement goes along with a much reduced sensitivity of the calculation on the assumptions regarding $b P(b)$. Furthermore, the experimental $4-\mathrm{D}$ plots for the transverse components are very similar to those for DI in $6 \mathrm{MeV} p+$ He collisions, corresponding to a very small $\eta$ of about 0.06 . The insensitivity to both $b P(b)$ and $\eta$ suggests that the 4-D plots for the transverse components are not very well suited to study the DI dynamics. On the other hand, the observation that theory is capable of reproducing the experimental plots, regardless of $\eta$, shows that in the transverse direction the 4-D plots offer a good test of the theoretical codes for errors. 


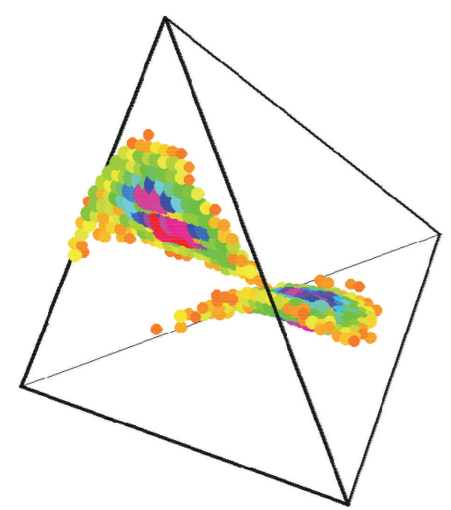

(a)

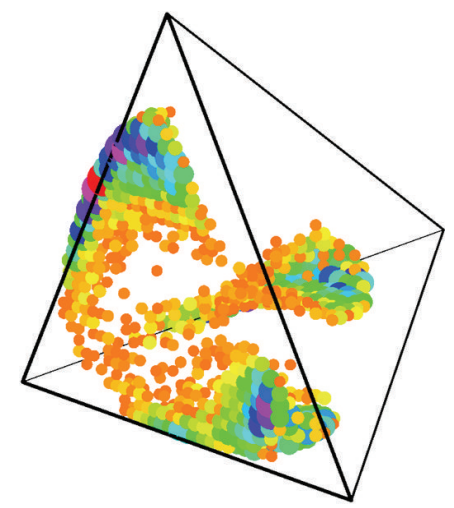

(c)

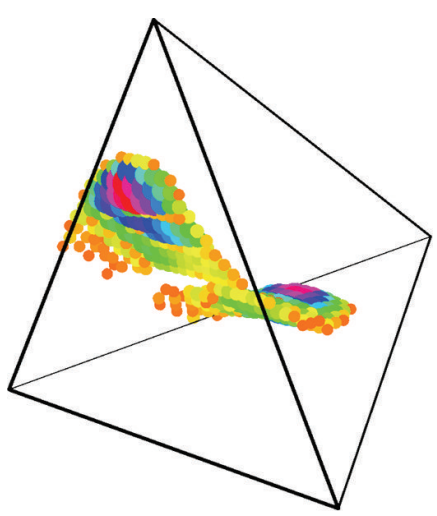

(b)

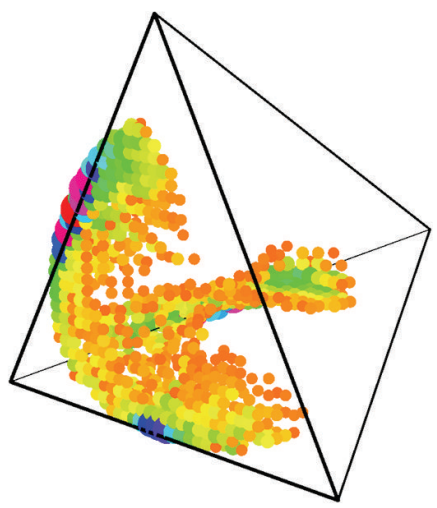

(d)

Figure 3: (Color online) Four body Dalitz plots (4-D) for DI in $158 \mathrm{MeV} \mathrm{Au}{ }^{33+}+\mathrm{He}$ collisions using the longitudinal momentum components of each particle. (a) experimental data, (b) TS-2 CDW-EIS, (c) TS-1 and (d) TS-2 FBA (see the text for details).

In contrast to the 4-D plots for the transverse components, those for the longitudinal components are very sensitive to the collision dynamics. Here, the plots look qualitatively very different for small and large $\eta$ [19]. After testing the codes using the transverse components, a comparison between experiment and theory using the longitudinal components should therefore provide detailed information about the DI dynamics. This comparison is shown in Figure 3 (experiment: panel (a); TS-2 CDW-EIS: panel (b)). It should be noted that in the longitudinal direction elastic scattering between the heavy particles has essentially no effect because the longitudinal momentum transfer is to a very good approximation directly proportional to the inelasticity. Qualitatively, the data are very well reproduced by the TS2 CDW-EIS calculation. In contrast, a TS-1 calculation based on the First Born Approximation (FBA) [18], shown in panel (c) of Figure 3, is in rather poor agreement with experiment. The TS-2 CDW-EIS and TS- 1 calculations shown in Figure 3 differ fundamentally from each other in two aspects: first, both calculations obviously treat different DI mechanisms. Second, the TS-2 CDW-EIS model treats already the ejection of a single electron to higher order, while the TS-1 model is a first-order calculation even for the complete DI process. A comparison between the TS- 1 results and a TS-2 calculation in which the ejection of each electron is treated within the FBA [2] (TS-2 FBA, panel d of Figure 3) shows that different DI mechanisms lead to nonnegligible differences in the longitudinal 4-D plots. However, earlier we demonstrated in detail that most of the characteristic features in the experimental plot can be explained by higher-order effects in the ejection of a single electron. More specifically, the postcollision interaction (PCI) between the outgoing projectile and one or both of the ejected electrons plays a very important role [2].

\section{Conclusions and Outlook}

We have presented a first attempt to model higher-order processes in double ionization of helium by highly charged particles. The approach treats double ionization as a sequential process where the projectile first ionizes a neutral helium atom and subsequently removes the remaining electron of the $\mathrm{He}^{+}$ion. Each step on this sequence is a single ionization process and can be modeled using the CDWEIS theory. We have compared our theoretical results on 4body Dalitz plots with the experimental data. These plots are generated using a theoretical event file, that was computed using the Monte Carlo Event Generator modified accordingly to consider double ionization as a sort of convolution of two independent single ionization steps. Good agreement with the experiments for the case of double ionization of helium by impact of $158 \mathrm{MeV}$ of $\mathrm{Au}^{33+}$ projectiles was 
observed in the 4-D plots generated for the longitudinal and transverse components. We have also proven that the 4 -D plots produced using the transverse components are quite insensitive to the charge to velocity ratio and in this way allow us to validate our different theoretical approaches. Among the next steps in order to overcome the remaining discrepancies, we plan to develop and implement a more formal theory for double ionization where both first and higher-order processes are treated coherently.

\section{References}

[1] J. Berakdar, A. Lahmam-Bennani, and C. Dal Cappello, "The electron-impact double ionization of atoms: an insight into the four-body coulomb scattering dynamics," Physics Report, vol. 374, no. 2, pp. 91-164, 2003.

[2] M. Schulz, M. F. Ciappina, T. Kirchner, D. Fischer, R. Moshammer, and J. Ullrich, "Role of elastic projectile-electron scattering in double ionization of helium by fast proton impact," Physical Review A, vol. 79, no. 4, Article ID 042708, 2009.

[3] M. Schulz, R. Moshammer, D. Fischer, et al., "Threedimensional imaging of atomic four-body processes," Nature, vol. 422, no. 6927, pp. 48-50, 2003.

[4] H. Ehrhardt, K. Jung, G. Knoth, and P. Schlemmer, "Differential cross sections of direct single electron impact ionization," Zeitschrift für Physik D, vol. 1, no. 1, pp. 3-32, 1986.

[5] M. Schulz and D. H. Madison, "Studies of the few-body problem in atomic break-up processes," International Journal of Modern Physics A, vol. 21, no. 18, pp. 3649-3672, 2006.

[6] I. Taouil, A. Lahmam-Bennani, A. Duguet, and L. Avaldi, "Fully determined (e, 3e) experiments for the double ionization of helium," Physical Review Letters, vol. 81, no. 21, pp. 4600-4603, 1998.

[7] A. Dorn, A. Kheifets, C. D. Schröter, et al., "Double ionization of helium by electron-impact: complete pictures of the fourbody breakup dynamics," Physical Review Letters, vol. 86, no. 17, pp. 3755-3758, 2001.

[8] D. Fischer, R. Moshammer, A. Dorn, et al., "Projectile-charge sign dependence of four-particle dynamics in helium double ionization," Physical Review Letters, vol. 90, no. 24, Article ID 243201, 2003

[9] M. Schulz, T. Ferger, D. Fischer, R. Moshammer, and J. Ullrich, "Multiple scattering mechanisms in simultaneous projectileelectron and target-electron ejection in $\mathrm{H}^{-}+\mathrm{He}$ collisions," Physical Review A, vol. 74, no. 4, Article ID 042705, 2006.

[10] J. Ullrich, R. Moshammer, R. Dörner, et al., "Recoil-ion momentum spectroscopy," Journal of Physics B, vol. 30, no. 13, pp. 2917-2974, 1997.

[11] M. Dürr, B. Najjari, M. Schulz, et al., "Analysis of experimental data for ion-impact single ionization of helium with Monte Carlo event generators based on quantum theory," Physical Review A, vol. 75, no. 6, Article ID 062708, 14 pages, 2007.

[12] M. Schulz, D. Fischer, T. Ferger, R. Moshammer, and J. Ullrich, "Four-particle dalitz plots to visualize atomic breakup processes," Journal of Physics B, vol. 40, no. 15, pp. 30913099, 2007.

[13] M. Schulz, M. Dürr, B. Najjari, R. Moshammer, and J. Ullrich, "Reconciliation of measured fully differential single ionization data with the first Born approximation convoluted with elastic scattering," Physical Review A, vol. 76, no. 3, Article ID 032712, 8 pages, 2007.
[14] A. C. Laforge, K. N. Egodapitiya, J. S. Alexander, et al., "Investigation of higher-order contributions to single ionization of atomic hydrogen by $75 \mathrm{keV}$ proton impact," Physics Review Letters, vol. 103, Article ID 053201, 2009.

[15] M. F. Ciappina, T. Kirchner, and M. Schulz, "Monte Carlo event generators in atomic collisions: a new tool to tackle the few-body dynamics," Computer Physics Communications, vol. 181 , no. 4, pp. 813-820, 2010.

[16] D. Belkić, I. Mančev, and J. Hanssen, "Four-body methods for high-energy ion-atom collisions," Reviews of Modern Physics, vol. 80, no. 1, pp. 249-341, 2008.

[17] D. S. F. Crothers and J. F. McCann, "Ionisation of atoms by ion impact," Journal of Physics B, vol. 16, no. 17, pp. 3229-3242, 1983.

[18] M. F. Ciappina, M. Schulz, T. Kirchner, D. Fischer, R. Moshammer, and J. Ullrich, "Double ionization of helium by ion impact analyzed using four-body Dalitz plots," Physical Review A, vol. 77, no. 6, Article ID 062706, 12 pages, 2008.

[19] D. Fischer, M. Schulz, K. Schneider, et al., "Systematic analysis of double-ionization dynamics based on four-body Dalitz plots," Physical Review A, vol. 80, no. 6, Article ID 062703, 8 pages, 2009.

[20] P. D. Fainstein, V. H. Ponce, and R. D. Rivarola, "Two-centre effects in ionization by ion impact," Journal of Physics B, vol. 24 , no. 14, p. 3091, 1991.

[21] N. Stolterfoht, R. D. DuBois, and R. D. Rivarola, Electron Emission in Heavy Ion-Atom Collisions, Springer, Berlin, Germany, 1997.

[22] E. Clementi and C. Roetti, "Roothaan Hartree Fock atomic wavefunctions. Basis functions and their coefficients for ground and certain excited states of neutral and ionized atoms, $\mathrm{Z} \leq 54$," Atomic Data and Nuclear Data Tables, vol. 14, no. 3-4, pp. 177-478, 1974.

[23] C. R. Garibotti and J. E. Miraglia, "Ionization and electron capture to the continuum in the $\mathrm{H}^{+}$-hydrogen-atom collision," Physical Review A, vol. 21, no. 2, pp. 572-580, 1980.

[24] D. S. F. Crothers and L. J. Dubé, "Continuum distorted wave methods in ion-atom collisions," Advances in Atomic, Molecular, and Optical Physics, vol. 30, pp. 287-337, 1993. 

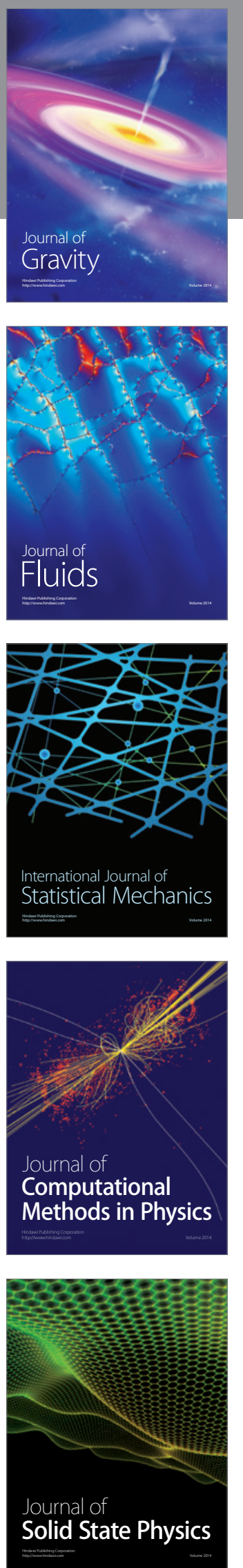

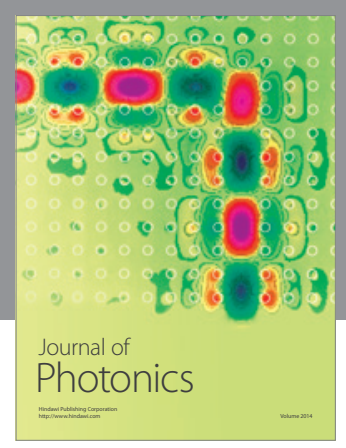

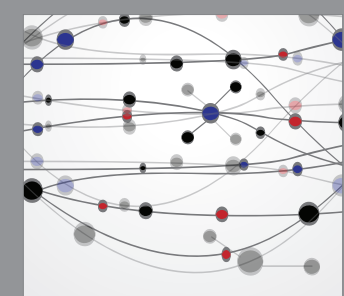

The Scientific World Journal
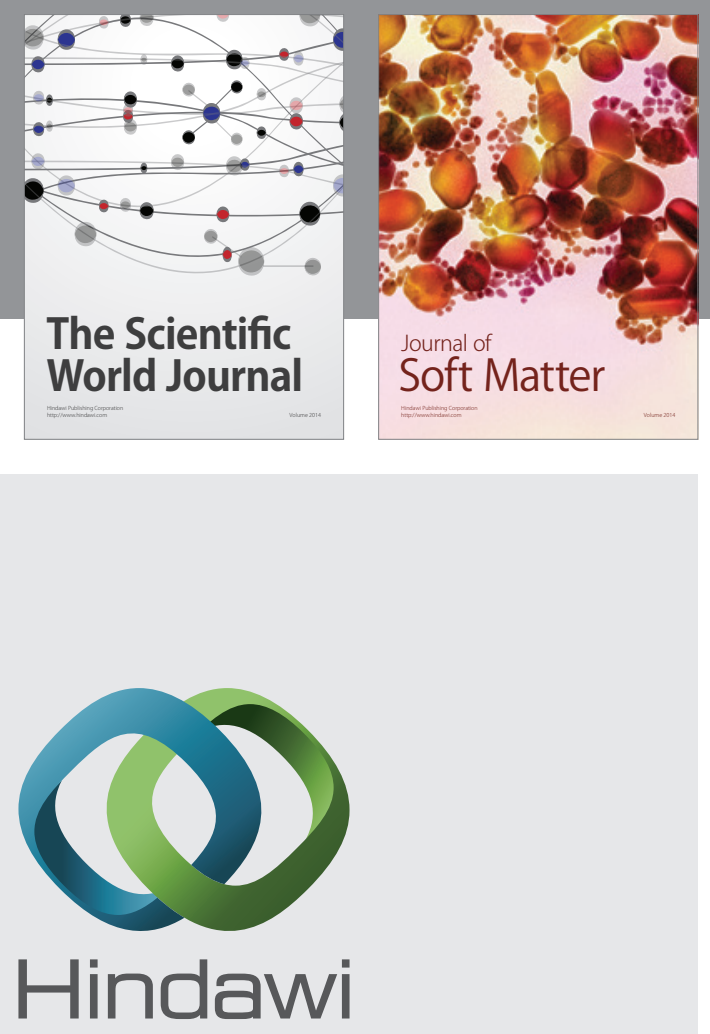

Submit your manuscripts at

http://www.hindawi.com
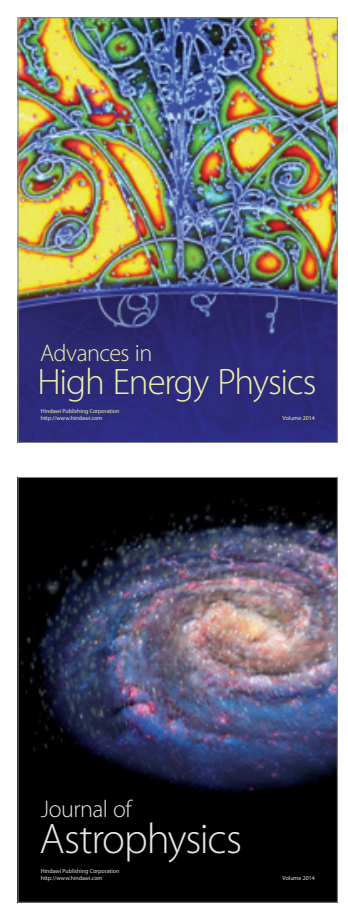
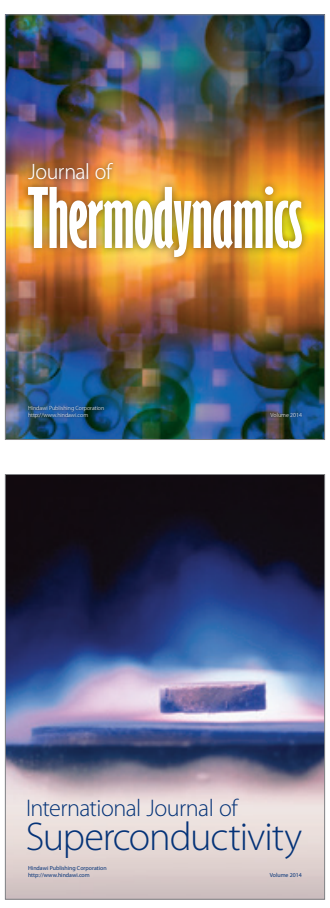
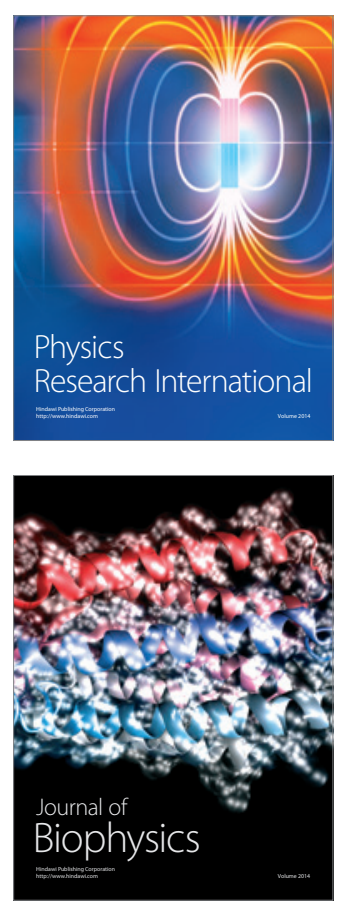
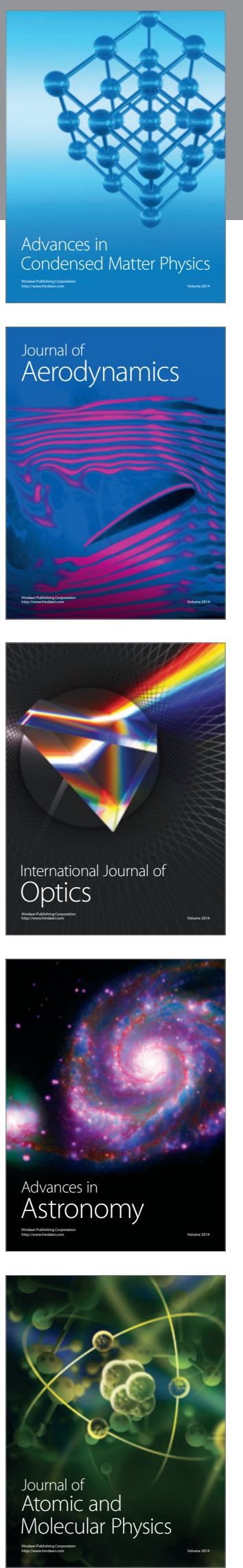